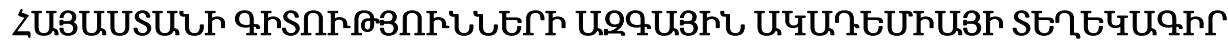 ИЗВЕСТИЯ НАЦИОНАЛЬНОЙ АКАДЕМИИ НАУК АРМЕНИИ
}

\begin{tabular}{|c|c|c|}
\hline Uthumuhlqu & 73, №3, 2020 & Механика \\
\hline УДК 539.3 & & Doi- http://doi.org/10.33018/73.3.3 \\
\hline
\end{tabular}

Ключевые слова: спеченный неупрочняемый материал, пористость, прессование, напряжённодеформированное состояние, компьютерное моделирование.

\section{Margaryan M.A.}

Computer modeling the process of pressing a round sintered sample in conical die Keywords: sintered material, porosity, pressing, stress-strain state, computer simulation

The process of pressing a round sintered sample in conical dies with different tapering was modeled in the ABAQUS software environment. On the upper butt-end of the cylindrical sample, displacement in the vertical direction is given, with which it passes through the container, the die, and calibration part of the press mould. The zones of the distribution of Mises stress, the main components of the stress state, the strain intensity and porosity of the material are obtained, from which the data in zone of the conical die are analyzed. The values of the components of the stress state and porosity are determined. The graphs of these quantities were plotted at the entrance to the die and at the exit from the die, as well as graphs of averaged data over the cross section along the die. Based on the obtained data, the features of the effect of the angle tapering of die on the pressing process are revealed and some assumptions used in solving the problem by the analytical method are confirmed.

Uupquipjuir U.U.

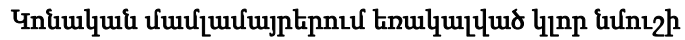

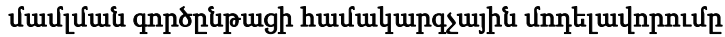

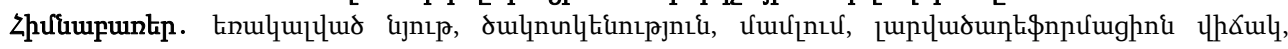

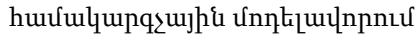

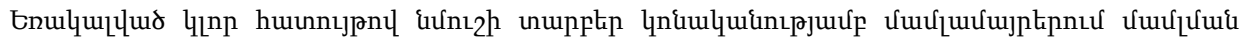

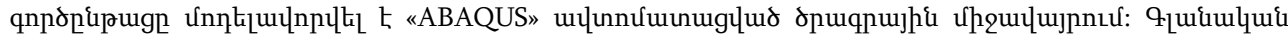

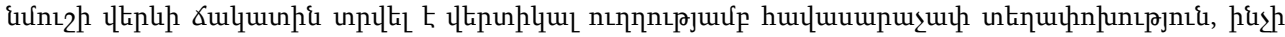

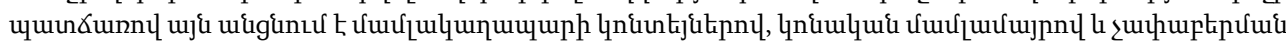

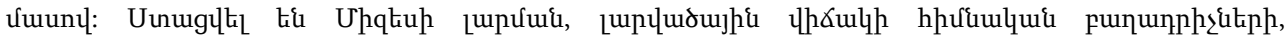

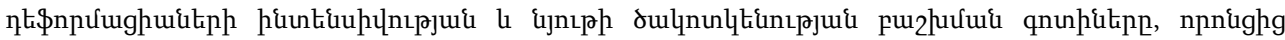

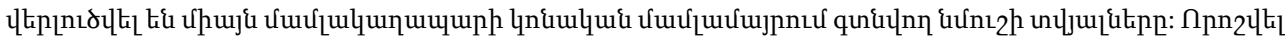

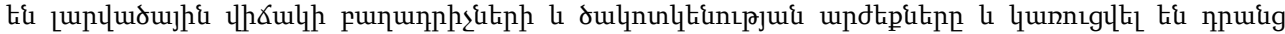

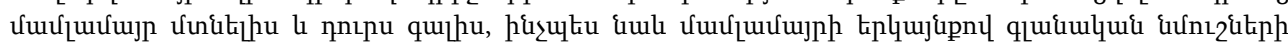

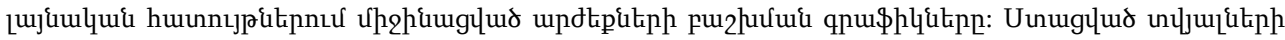

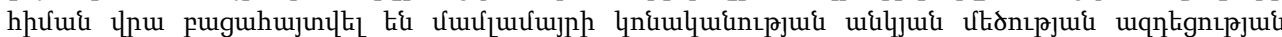

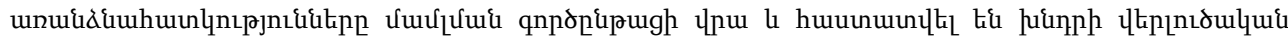

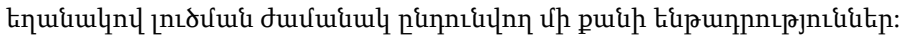

В программной среде «ABAQUS» смоделирован процесс прессования спечённого образца круглого сечения в конических матрицах различной конусности. К его верхнему торцу подаётся равномерное перемещение в вертикальном направлении, в результате чего образец проходит через контейнер, коническую матрицу и калибровочную части пресс-формы. Получены зоны распределения напряжения Мизеса, основных компонентов напряжённого состояния, интенсивности деформаций и пористости материала, на основе которых были проанализированы только данные для образца, находящегося в конической матрице. Определены значения компонентов напряжённого состояния и пористости, построены 44 
графики этих величин при входе в матрицу и на выходе из неё, а также графики усреднённых данных по поперечному сечению вдоль матрицы. На основе полученных данных выявлены особенности влияния величины угла конусности матрицы на процесс прессования и подтверждены некоторые допущения, используемые при решении задачи аналитическим методом.

Моделирование инженерных задач с использованием автоматизированных программных сред (АПС) позволяет в течение короткого времени получать результаты с достаточно большой точностью. При этом, возникаемые проблемы могут решаться как в области упругих деформаций (расчёт прочности), так и в области пластических деформаций (технологический расчёт). Целесообразность использования АПС подтверждена по результатам многочисленных научно-практических работ. В частности, можно отметить работы, проведённые в среде «ABAQUS» $[1,2]$. Иногда, в зависимости от типа задач, в их решениях используются методы сопоставления аналитического метода и АПС или сопоставления двух разных АПС [3].

Исследования процессов прессования стержней из спечённого материала аналитическим методом достаточно сложны и мало изучены из-за наличия пористости в материале [4-6]. Что касается компьютерного моделирования этих задач для матриц различной конусности, то таковое практически не применялось, следовательно, работы в этом направлении актуальны.

Цель работы - в автоматизированной программной среде «ABAQUS» исследование распределения напряжённо-деформированного состояния и пористости спечённых стержней при прессовании в матрицах различной конусности и выявление влияния угла конусности на параметры технологического процесса.

На фиг. 1 в трёх частях пресс-формы (контейнере, конической матрице и калибровочной части) показаны зоны перемещения в горизонтальном и вертикальном направлениях цилиндрического образца, полученные путём подачи равномерного перемещения со стороны верхнего торца.

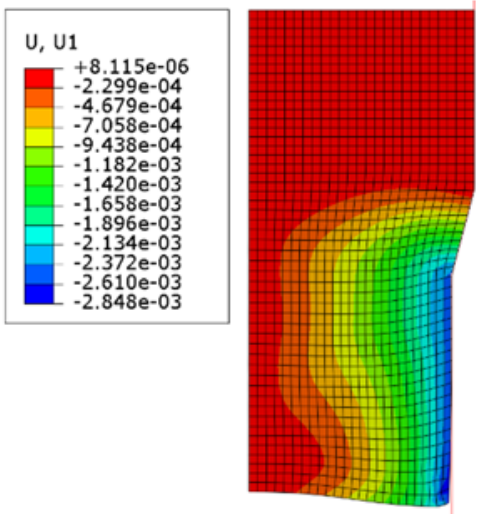

a)

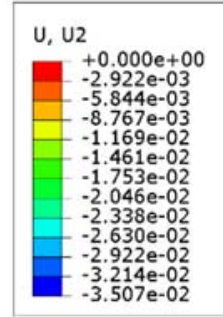

б)

Фиг. 1. Зоны перемещения, полученные в АПС в горизонтальном (а) и вертикальном (б) направлениях

Моделирование задачи в АПC «ABAQUS». Начальные условия задачи: начальный и конечный (готовое изделие) диаметры стержня - $\mathrm{D}_{0}=25$ мм и $\mathrm{d}=22,5$ мм, коэффициент контактного трения $-f=0,1$, углы конусности матрицы $-\varphi=$ 
$15^{0}, 20^{0}, 30^{0}$, тип четырёхугольных элементов сети при делении стержня конечным числом элементов - CAX4R, количество - 1250 .

Параметры материала: плотность -8000 к2/ $\mathrm{M}^{3}$, относительная плотность $-0,9$ (в соответствии с которой начальная пористость материала $\left.-v_{0}=0,1\right)$, модуль Юнга - 210 ГПа, коэффициент Пуассона - 0,3. Для решения задачи был выбран неупрочняемый материал с пределом текучести $\sigma_{T}=350$ МПа.

В результате решения задачи были получены все компоненты напряжённодеформированного состояния. На фиг. 2-8 на половине осевого сечения цилиндра показаны зоны распределения характерных величин его напряжённо-деформационного состояния: интенсивностей напряжений $\sigma_{i}$ (напряжения Мизеса) и деформаций $\varepsilon_{i}$ [7], компонентов напряжённого состояния (радиальных $\mathrm{S}_{11}=\sigma_{r}$, окружных $\mathrm{S}_{33}=\sigma_{\theta}$, осевых $\mathrm{S}_{22}=\sigma_{z}$ и касательных $\mathrm{S}_{12}=\tau_{r z}$ напряжений) и пористости материала $v$.

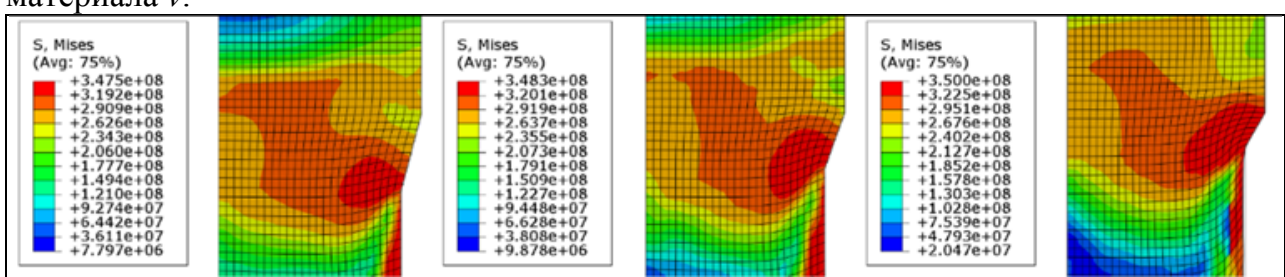

Фиг. 2. Напряжения Мизеса при $\varphi=15^{\circ}, 20^{\circ}, 30^{\circ}$ в $A \Pi C$ «ABAQUS»

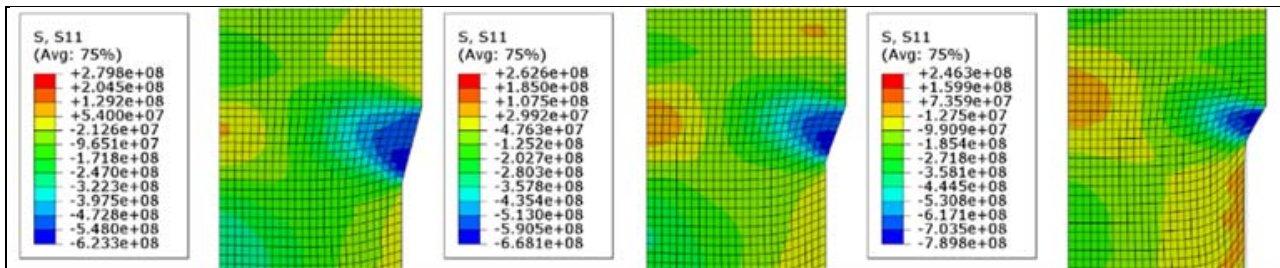

Фиг.3. Зоны радиальных $\sigma_{\mathrm{r}}$ напряжений при $\varphi=15^{\circ}, 20^{\circ}, 30^{\circ}$ в $A \Pi C$ «ABAQUS»

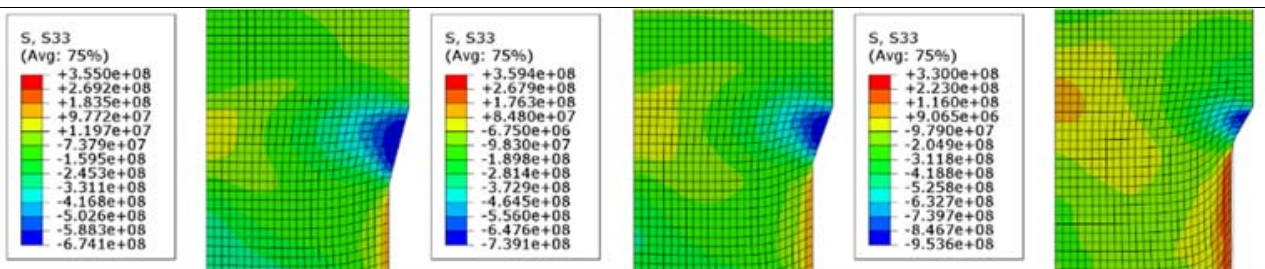

Фиг.4. Зоны окружных $\sigma_{\theta}$ напряжений при $\varphi=15^{\circ}, 20^{\circ}, 30^{\circ}$ в AПC «ABAQUS»

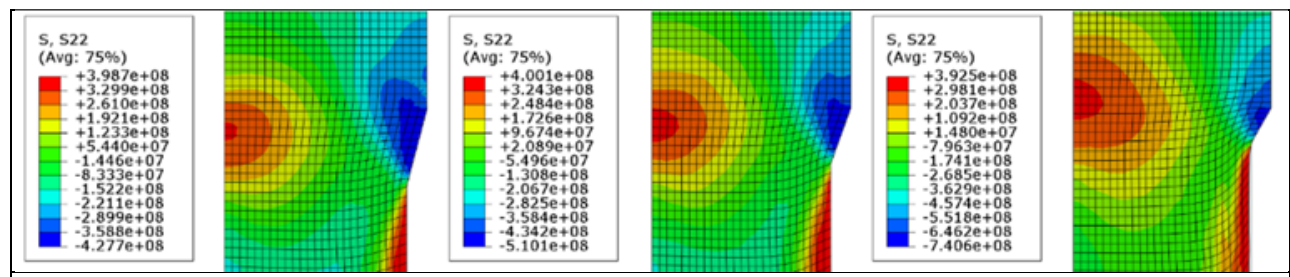

Фиг.5. Зоны осевых $\sigma_{\mathrm{z}}$ напряжений при $\varphi=15^{\circ}, 20^{\circ}, 30^{\circ}$ в AПC «ABAQUS» 

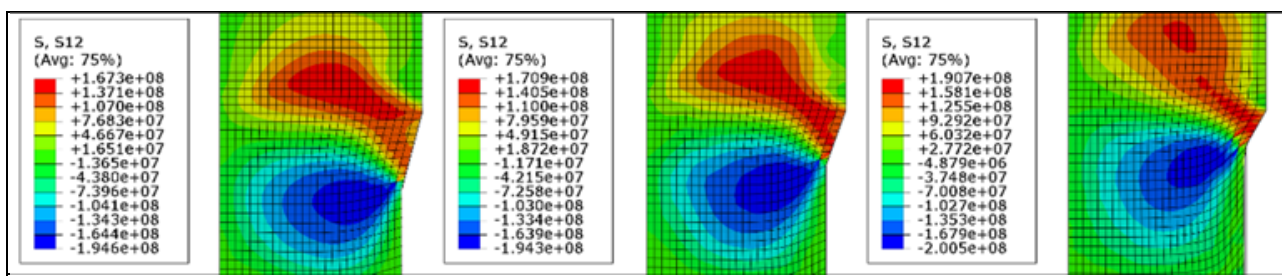

Фиг.6. . Зоны касательных $\tau_{\mathrm{rz}}$ напряжений при $\varphi=15^{\circ}, 20^{\circ}, 30^{\circ}$ в $A \Pi C$ «ABAQUS»

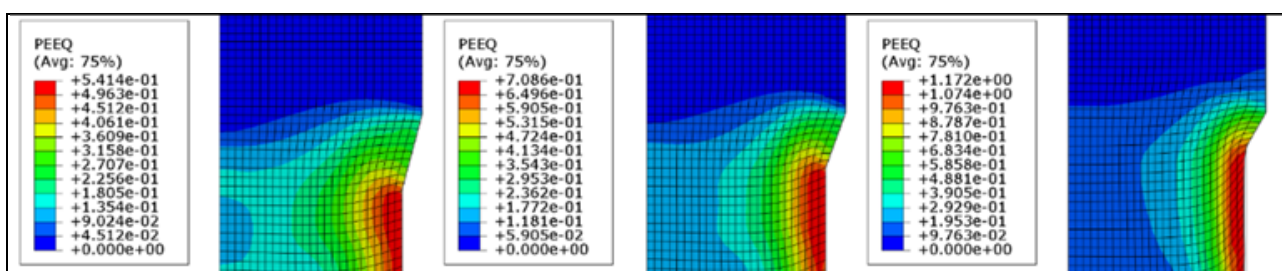

Фиг.7. . Зоны интенсивности деформаций $\varepsilon_{\mathrm{i}}$ при $\varphi=15^{\circ}, 20^{\circ}, 30^{\circ}$ в АПС «ABAQUS»

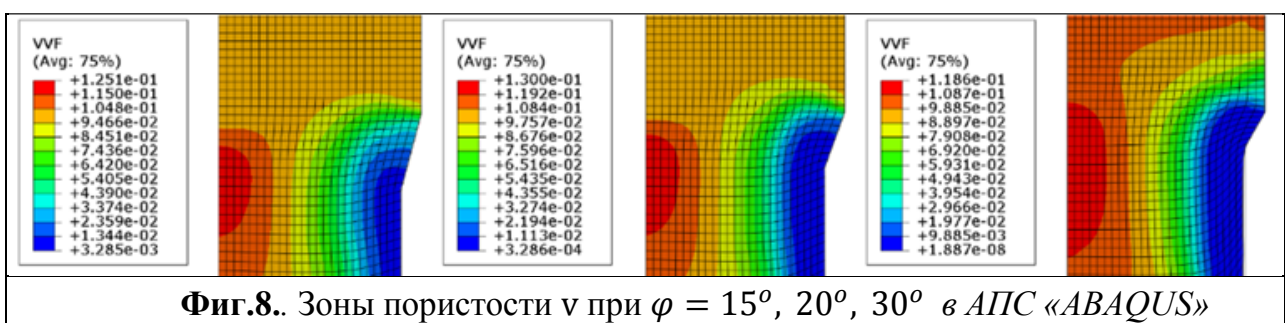

На фиг. 9-11 представлены графики распределения величин нормальных напряжений (величины касательных напряжений не обсуждаются, так как они в несколько раз меньше нормальных) в АПС при входе в матрицу и на выходе из неё (точка 0 находится на оси образца, а точка 25 - на его краевом контуре).

В результате исследования графиков, полученных на фиг 9-11, можно сделать следующие выводы: напряжения $\sigma_{\theta}$ и $\sigma_{\mathrm{r}}$ почти одинаковы (что принимается при решении проблемы аналитическим методом [4,8,9]); абсолютные значения напряжений при входе в матрицу значительно выше по сравнению с напряжениями при выходе из матрицы; во всех точках контакта с матрицы все напряжения отрицательные, а на оси - в основном, положительные.

На фиг.12 представлены графики распределения усреднённых значений компонентов напряжённого состояния в поперечных сечениях цилиндрических образцов - вдоль матрицы. 


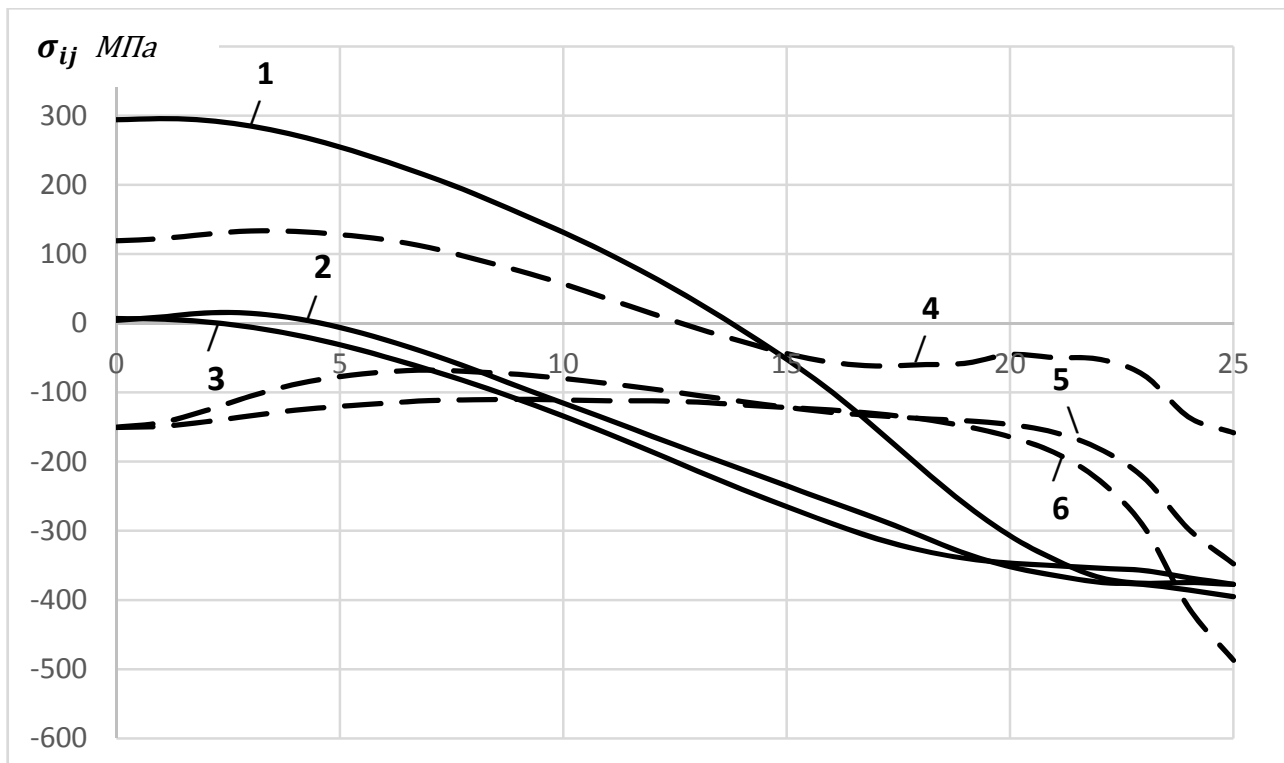

Фиг.9. Кривые компонентов напряжения при входе в матрицу

$\left(1-\sigma_{z}, 2-\sigma_{r}, 3-\sigma_{\theta}\right)$ и на выходе из неё $\left(4-\sigma_{z}, 5-\sigma_{r}, 6-\sigma_{\theta}\right)$ при $\varphi=15^{\circ}$

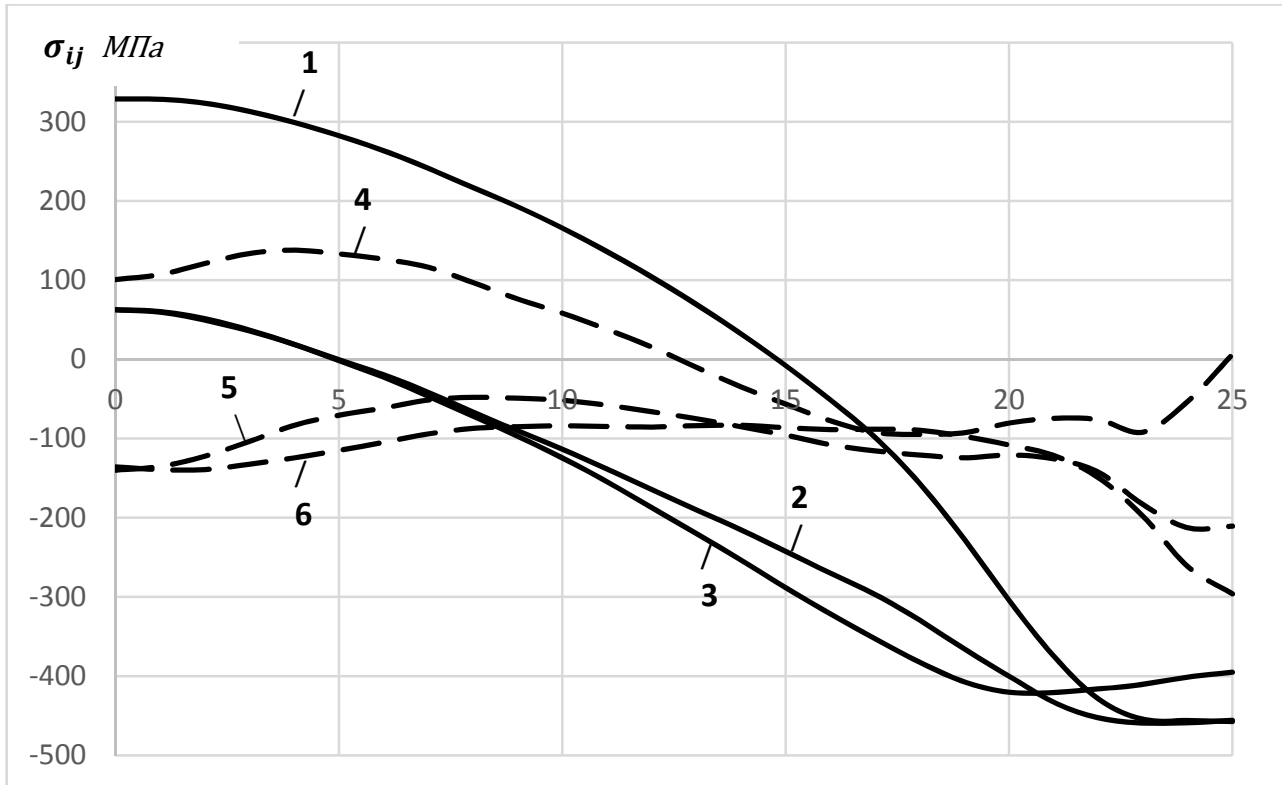

Фиг.10. Кривые компонентов напряжения при входе в матрицу

$\left(1-\sigma_{z}, 2-\sigma_{r}, 3-\sigma_{\theta}\right)$ и на выходе из неё $\left(4-\sigma_{z}, 5-\sigma_{r}, 6-\sigma_{\theta}\right)$ при $\varphi=20^{\circ}$ 


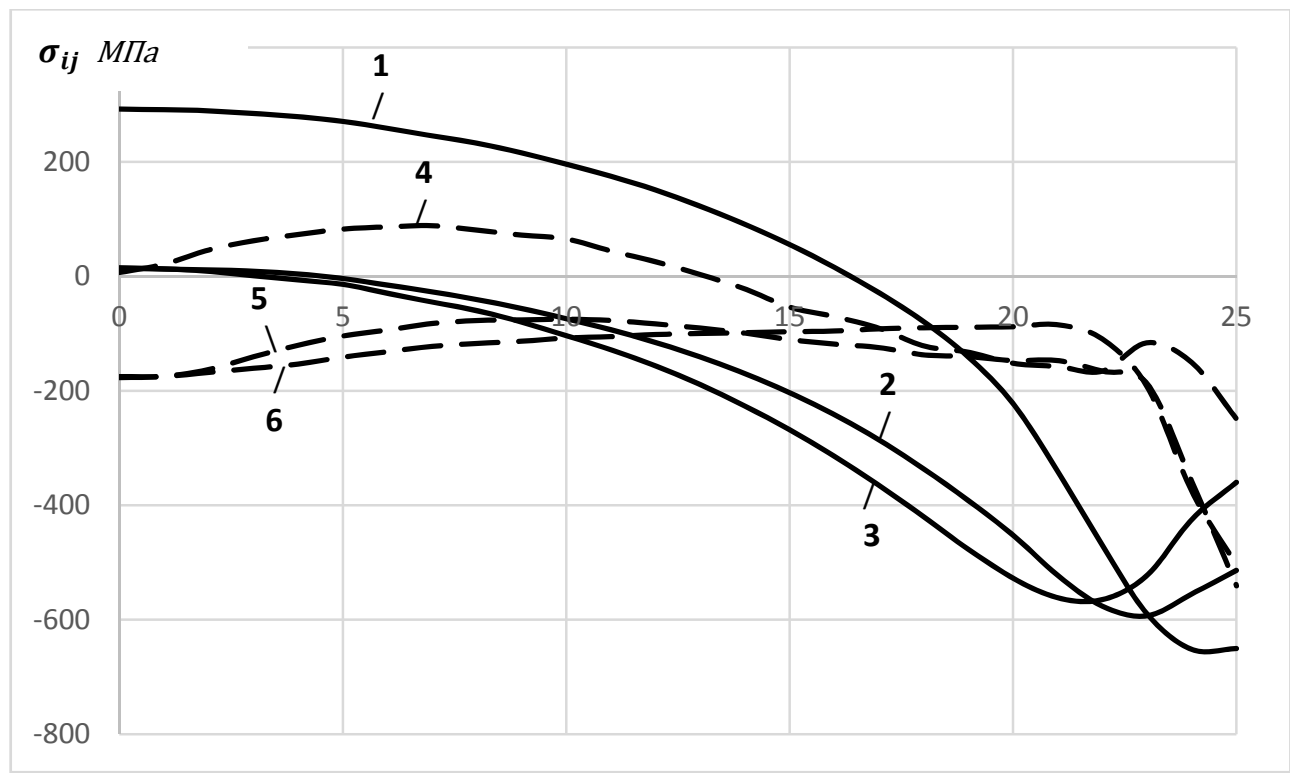

Фиг.11. Кривые компонентов напряжения при входе в матрицу

$\left(1-\sigma_{z}, 2-\sigma_{r}, 3-\sigma_{\theta}\right)$ и на выходе из неё $\left(4-\sigma_{z}, 5-\sigma_{r}, 6-\sigma_{\theta}\right)$ при $\varphi=30^{\circ}$

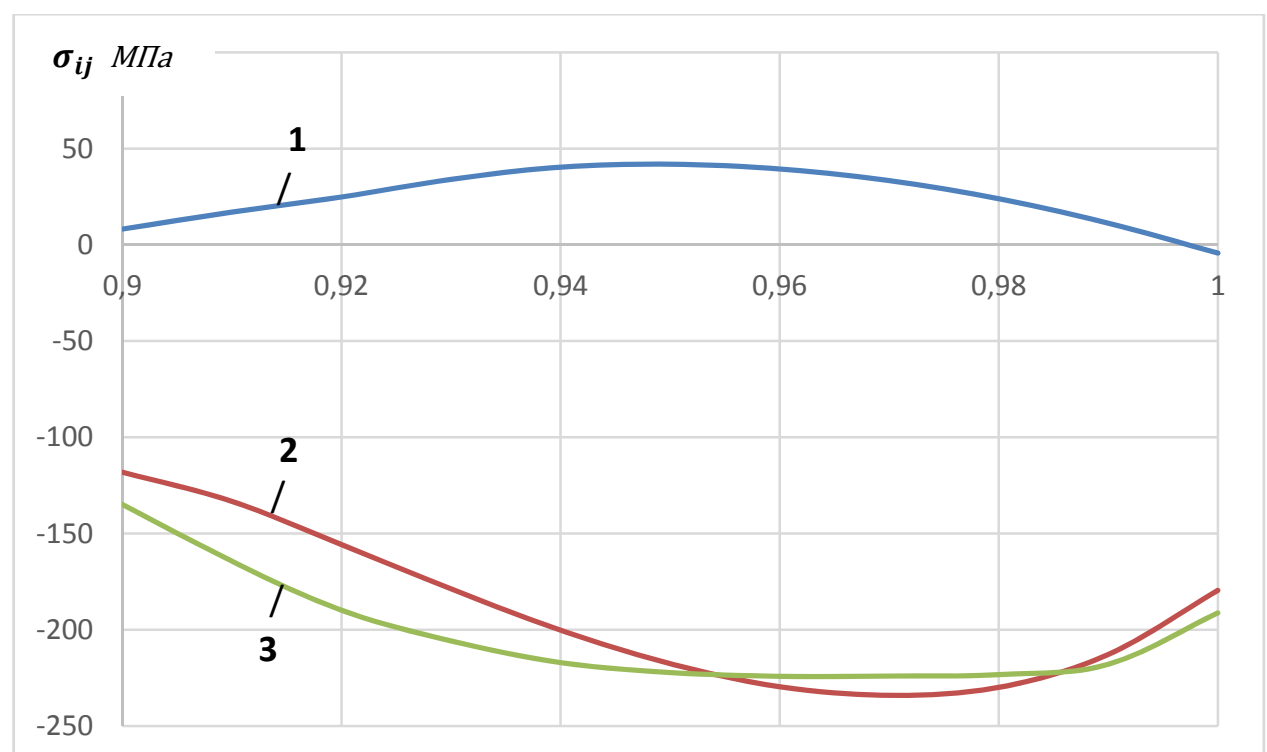

Фиг. 12. Усреднённые значения напряжённого состояния $\sigma_{z}(1), \sigma_{\theta}(2)$ и $\sigma_{r}(3)$ в поперечных сечениях цилиндрических образцов вдоль матрицы при $\varphi=15^{\circ}$

Как видно из фиг. 12 , в случае $\varphi=15^{\circ}$ максимальные значения сжатия напряжений $\sigma_{\theta} \approx \sigma_{\mathrm{r}}$ получаются на отрезке $\sim \mathrm{r} / \mathrm{r}_{0}=0,97$, то есть в сечениях, близко расположенных к входу конической матрицы, что совпадает с данными [4]. 
Что касается данных кривой 1 осевых напряжений $\sigma_{z}$ (рис. 12), построенной по таблице, то причиной ее малых положительных величин является неравномерное распределение $\sigma_{\mathrm{z}}$ в поперечном сечении (рис. 9-11). Это результат следующего основного граничного условия деформирования образца: к его верхнему торцу подается равномерное перемещение $u_{2}$ (рис. 16). В этом случае в образце сжимающие осевые напряжения возникают только в контактных с матрицей слоях.

\section{Таблица}

Данные $\sigma_{z}$ (в МПа) вдоль высоты матрицы в шести линиях сетки конечных элементов по радиусу от оси образца

\begin{tabular}{|c|c|c|c|c|c|c|c|}
\hline $\begin{array}{r}\text { Номера } \\
\text { линии } \\
\text { точек }\end{array}$ & 1 & 2 & 3 & 4 & 5 & 6 & $\begin{array}{c}\text { Усредненные } \\
\text { значения, Мпа }\end{array}$ \\
\hline 1 & 294 & 255 & 131 & -52 & -307 & -378 & $-9,5$ \\
\hline 2 & 325 & 272 & 145 & -31 & -291 & -393 & 4,5 \\
\hline 3 & 340 & 281 & 155 & -13 & -267 & -395 & 16,8 \\
\hline 4 & 337 & 283 & 158 & 2 & -230 & -402 & 24,7 \\
\hline 5 & 324 & 276 & 155 & 10 & -176 & -409 & 30 \\
\hline 6 & 307 & 262 & 145 & 12 & -115 & -417 & 32,3 \\
\hline 7 & 279 & 237 & 129 & 5 & -71 & -423 & 26 \\
\hline 8 & 234 & 205 & 108 & -8 & -47 & -428 & 10,7 \\
\hline
\end{tabular}

На фиг. 13-16 показаны кривые распределения пористости в АПС при входе в матрицу и на выходе из неё и усреднённые значения пористости вдоль матрицы при разных $\varphi$.

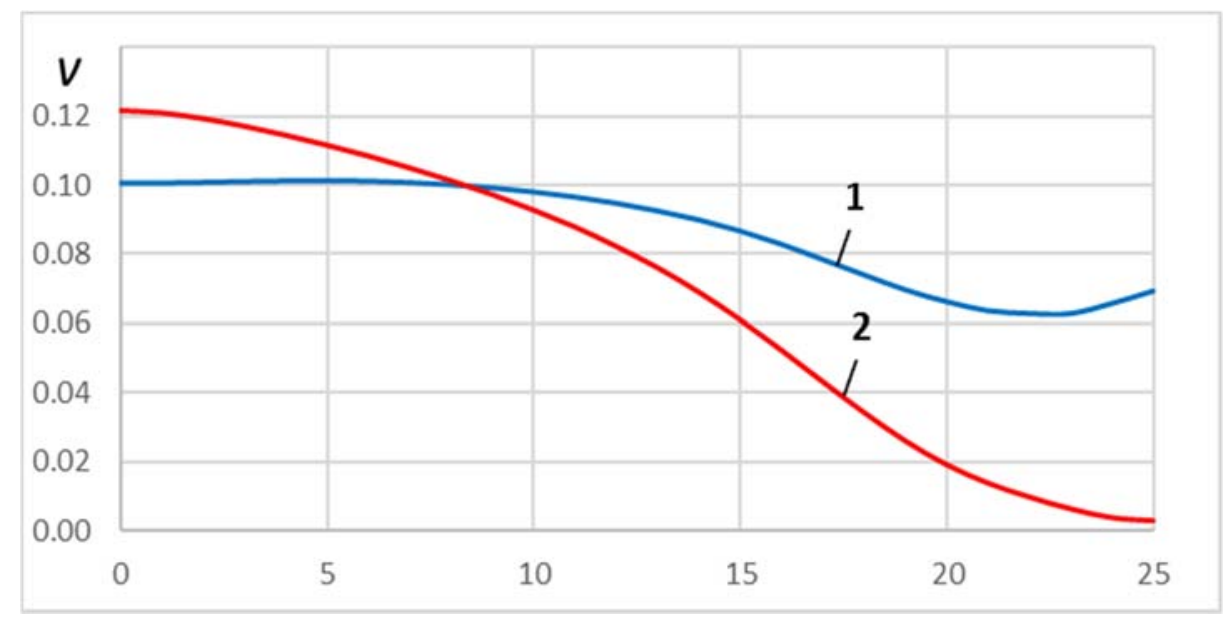

Рис. 13. Значения пористости v в поперечном сечении при входе в матрицу (1) и на выходе из нее (2) при $\varphi=15^{\circ}$ 


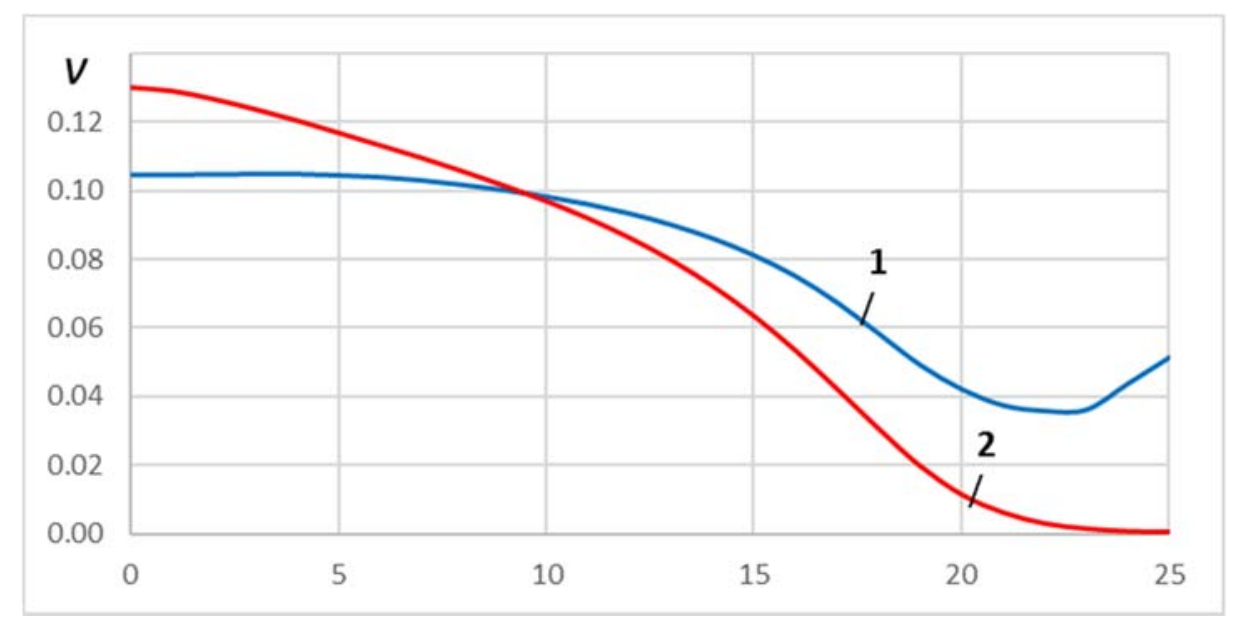

Рис. 14. Изменение значения пористости v при $\varphi=20^{\circ}$

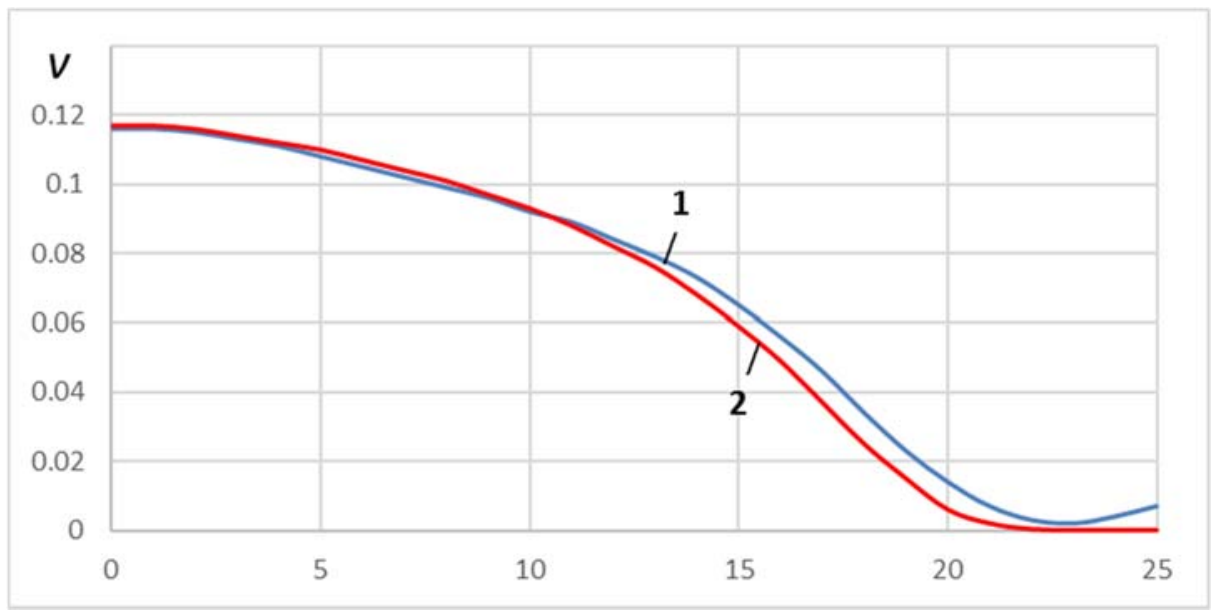

Рис. 15. Изменение значения пористости $v$ при $\varphi=30^{\circ}$

Сравнительные данные, приведённые на фиг. 13-15, показывают, что уплотнение образцов начинается с контактирующих с матрицей слоёв, причём при $\varphi=15^{\circ}$ на выходе из матрицы эти слои полностью уплотняются, при вхождении в матрицу в центральной части материала пористость не меняется, а при выходе из нее - она увеличивается. При $\varphi=20^{\circ}$ на входе в матрицу пористость материала начинает увеличиваться, при $\varphi=30^{\circ}$ в результате процесса прессования наружная поверхность материала полностью уплотняется, а в центре пористость увеличивается, и кривые 1 и 2 совпадают. Фактически, при прессовании образца с начальной пористостью $\mathrm{v}_{0}=10 \%$ в матрице с конусностью $\varphi=30^{\circ}$, образец превращается в стержень с переменной пористостью в зависимости от его радиуса. 


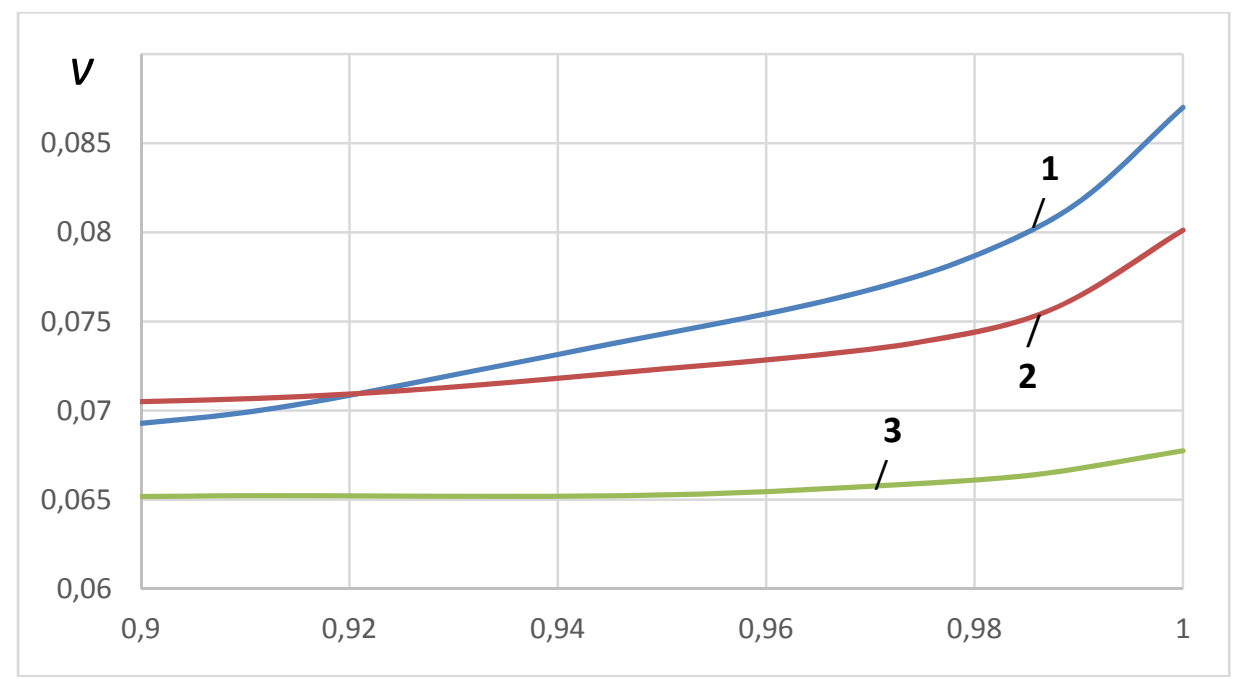

Рис. 16. Усредненные значения распределения пористости вдоль матрицы при $\mathrm{v}_{0}=0,1, \varphi=15^{\circ}(1), 20^{\circ}(2), 30^{\circ}(3)$

Из фиг. 16 видно, что при углах $15^{\circ}$ и $20^{\circ}$ значения усреднённой пористости при входе в матрицу $\left(v_{1}\right)$ и на выходе и неё $\left(v_{2}\right)$ достаточно различаются $\left(v_{l}>v_{2}\right)$, а при $30^{\circ}$ они почти совпадают $\left(v_{1} \approx v_{2}\right)$, то есть получается примерно равномерно распределённая вдоль деформированного образца усреднённая пористость и $v_{3}=6,5 \%$.

Таким образом, при значениях угла конусности $\varphi=15^{0}, 20^{\circ}, 30^{\circ}$ в АПС «ABAQUS» определены значения компонентов напряжённо-деформированного состояния образца и величины распределения пористости при входе в матрицу и на выходе из неё. На основе полученных данных построены соответствующие кривые и проведён анализ полученных результатов.

\section{ЗАКЛЮЧЕНИЕ}

В автоматизированной программной среде «ABAQUS» выполнено моделирование компонентов напряжённо-деформированного состояния и величин распределения пористости в процессе прессования спечённого цилиндрического образца при различных углах конусности матрицы.

Показано, что напряжения $\sigma_{\theta}$ и $\sigma_{\mathrm{r}}$ почти одинаковы, в контактных слоях образца по всей протяжённости матрицы имеются достаточно большие сжимающие напряжения, и при входе в матрицу абсолютные значения напряжения намного больше, чем на выходе из неё. При $\varphi=15^{\circ}$ максимальные значения сжатия напряжений $\sigma_{\theta} \approx \sigma_{\mathrm{r}}$ получаются на близлежащих к входу конической матрицы участках, что совпадает с имеющимися в литературе данными.

Полученные значения изменения пористости свидетельствуют о том, что пористость в поперечном сечении образца распределяется неравномерно: при угле конусности $\varphi=30^{\circ}$ и начальной пористости материала $\mathrm{v}_{0}=10 \%$ в результате прессования внешняя поверхность образца полностью уплотняется - $\mathrm{V} \approx 0$, а в центре пористость увеличивается - $\mathrm{v} \approx 11,8 \%$.

Анализ распределения усреднённой пористости вдоль конической матрицы в деформированном образце при $\varphi=15^{0}, 20^{\circ}, 30^{0}$ показал, что в случае $\varphi=30^{\circ}$ 52 
получается примерно равномерное распределение средней пористости в деформированном образце - вдоль матрицы и на выходе из матрицы $v_{3}=6,5 \%$.

\section{ЛИТЕРАТУРА}

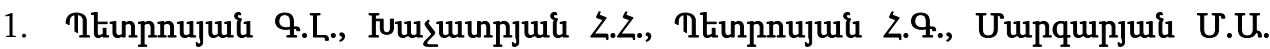

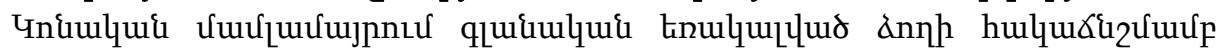

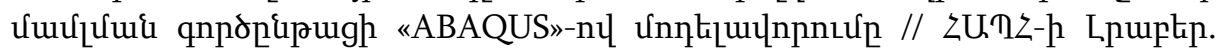

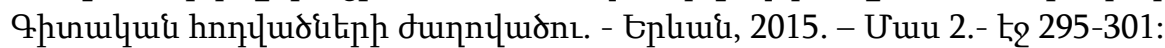

2. Петросян Г.Л., Хачатрян Г.Г., Сафарян М.Б., Маргарян М.А., Петросян А.Г. Моделирование процесса прессования круглого стержня в конической матрице в условиях больших противодавлений // Вестник НПУА: Механика, машиноведение, машиностроение.- Ереван, 2015.- N1. - С. 26-35.

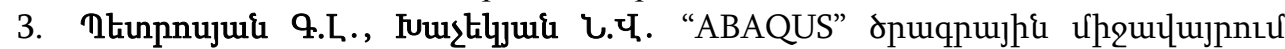

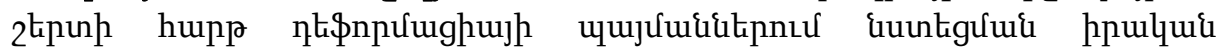

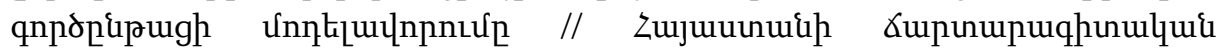

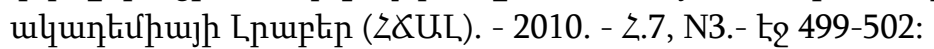

4. Петросян Г.Л. Пластическое деформирование порошковых материалов. М.: Металлургия, 1988. $153 \mathrm{c}$.

5. Петросян Г.Л., Мусаелян Г.В. Исследование влияния схемы деформирования на уплотняемость спеченных цилиндрических заготовок // Известия вузов. Машиностроение. 1983. №10. С. 102-105.

6. Петросян Г.Л., Мусаелян Г.В. Напряженное состояние пористых осесимметричных заготовок при выдавливании // Порошковая металлургия. 1984. №11. C. 32-35.

7. Малинин Н.Н. Прикладная теория пластичности и ползучести. М.: Машиностроение, 1975. 399с.

8. Томсен Э., Янг Ч., Кобаяши Ш. Механика пластических деформаций при обработке металлов. М.: Машиностроение, 1969. 504c.

9. Hosford W.F., Caddell R.M. Metal forming mechanics and metallurgy - Cambridge, 2007. 312p.

\section{Сведения об авторе:}

Маргарян Мигран Арнакович - аспирант Национального политехнического университета Армении, Институт механико-машиностроения, транспортных систем и дизайна.

Тел.: (+374 94) 1074 36; E-mail: marg.mihran@gmail.com

Поступила в редакцию 